\title{
Nanomaterial Fused Monolithic Microcolumns for Biomolecule Separation
}

\author{
M. A. Vijayalakshmi and Kishore K. R. Tetala* \\ Advanced Centre for Bioseparation Technology, Vellore Institute of Technology, Vellore, \\ Tamil Nadu-632014, India \\ Email: kishore.tetala@gmail.com
}

\begin{abstract}
Achieving a highly pure biomolecule of interest is of great importance and chromatography based techniques are the reliable tools for this challenging task. During the last few decades, monoliths have been explored as alternative chromatography stationary phases for biomolecule separation. In this Minireview, we aim to provide information on different nanomaterials incorporated within the network of monoliths and strategies applied to perform nanomaterial surface functionalization. Subsequently, the applications of "nanomaterials embedded monolithic microcolumns" for separation of target biomolecules such as proteins and peptides with the help of different chromatography principles such as affinity, ionic and hydrophobic is also discussed in detail.
\end{abstract}

Keywords monolith, nanomaterial, proteins, peptides, separation

\section{Introduction}

Chromatography based approaches are widely employed both in academia and industry for selective target biomolecule (proteins, peptides, carbohydrates, etc.) separation from other interfering molecules present in serum, plant extracts, etc. ${ }^{[1-4]}$ However, the technical requirements (tedious packing processes, high back pressures, and the need of frits to hold the resin within the column) of conventional chromatography resins limit their incorporation into microcolumns. ${ }^{[4]}$ In late 1980's and early 1990's, the research outcome of two research groups (Prof. Hjertén, ${ }^{[5]}$ Prof. Švec and Prof. Fréchet ${ }^{[6]}$ ) resulted in a new stationary phase material, christened as MONOLITHS (due to its physical nature, i.e., "a single piece of continuous interconnected porous network").

In general, monoliths are classified into two types (organic/ polymer monoliths and inorganic monoliths) depending on the differences such as molecules required, preparation approach, and physical properties (porosity, surface area and mechanical strength). ${ }^{[7-15]}$ Polymer monoliths require monomers, crosslinkers, porogen, and an initiator to initiate the in situ polymerization by heat, UV, or $\gamma$-rays. Methacrylate based polymers, polystyrenes, and agarose fall under the category of polymer monoliths. For example, methacrylate based monoliths in various formats (rods and disk) are commercially available under the trade name "CIM" (Convective Interaction Media) supplied by BIA separation (Slovenia). In case of inorganic monoliths, sol-gel technology is employed. For example, silica based monoliths are commercially available under the trade name "Chromoliths" from MERCK. The advantage of a typical monolith is to provide high mass transfer via convective flow that leads to rapid separation with high resolution, low pressure drops at higher flow rates, functional groups $\left(\mathrm{COOH}, \mathrm{NH}_{2}, \mathrm{OH}\right.$, etc.) on the surface for immobilization of desired ligands. Apart from these, the pre-polymer in the form of solution can easily be incorporated into microcolumns due to in situ polymerization possibility and does not require frits to hold the material within the microcolumn due to covalent adhesion of material with the column inner wall surface.
Since early 2000's, incorporation of nanomaterials within the confinements of monolithic network was initiated to further enhance the separation performance of the monoliths. ${ }^{[16-23]}$ The addition of nanomaterials was achieved either prior to monolith in situ polymerization or immobilized after the monolith is formed, i.e., form chemical bonds with the functional groups present on the monolith surface. The rationale to choose nanomaterials as anchors is due to their impressive inherent physical and chemical properties that enhance the separation performance of the monoliths.

The major objective of this Minireview is to bring out the different nanomaterials incorporated within the monolithic structures and to highlight the biomolecule separation achieved by these new hybrid materials. Table 1 presents the nanomaterials incorporated within monoliths and the ligands used for separation of target analytes.

\section{Gold nanoparticles (Au NP)}

The group of Prof. Švec and Prof. Fréchet has extensively worked on various approaches to incorporate Au NP's within the monolith matrix. In their first study, ${ }^{[24]}$ Au NP's (15 nm) were immobilized homogeneously on thiol functionalized poly GMAEDMA (glycidyl methacrylate-co-ethylene dimethacrylate) monolith and were used as ligands to target cysteine functionalized peptides. The adsorption capacity of this monolith for $L$-cysteine, the model analyte, was found to be $2.50 \mu \mathrm{moL}$ monolith. The monolith adsorbed selectively "His-Cys-Lys-PheTrp-Trp", i.e., cysteine containing peptide only and not noncysteine peptides (Tyr-Gly, Phe-Gly-Phe-Gly, and Tyr-Gly-GlyPhe-Leu). Although the Au NP monolith has the desired affinity property, it lacked the required hydrophobicity like a conventional reverse phase column to separate peptides. This issue was addressed by fusing the Au NP monolith column with a commercial C18 column (Acclaim PepMap) in a tandem fashion. This strategy has led to an efficient capture of cysteine peptides via Au NP monolith while non-cysteine peptides passed freely and retained by $\mathrm{C} 18$ column. In the second study, ${ }^{[25]}$ they introduced various functional groups on Au NP's present on 
Minireview

Table 1 Nanomaterials and ligands used for biomolecule separation

\begin{tabular}{|c|c|c|c|}
\hline Nanomaterials & Ligands & Analytes & Ref. \\
\hline $\mathrm{Au} N \mathrm{NP}$ & $\mathrm{Au}$ & Cysteine functional peptides & [24] \\
\hline Au NP & Various ligands & Peptides and proteins & [25] \\
\hline Au NP & 1-Octanethiol \& 1-octadecanethiol & Proteins & [26] \\
\hline Au NP & Cysteine & Peptides and nucleosides & [27] \\
\hline Au NP & Erythrina cristagalli & Glycoproteins & [28] \\
\hline Au NP & 1-Methyl-2-mercapto-3-butylimidazolium bromide & Nucleosides and nucleic acid bases & [29] \\
\hline Au NP & Cysteine & Glycopeptides & [30] \\
\hline Au NP & Human $\alpha$-thrombin aptamer & Human $\alpha$-thrombin & [31] \\
\hline Au NP & $\mathrm{Au}$ & Proteins & [32] \\
\hline Iron oxide NP & Iron oxide & Phosphopeptides & [33] \\
\hline SWNT & SWNT & Peptides & [34] \\
\hline MWNT & MWNT & Proteins & [35] \\
\hline MWNT & MWNT-C18 & Proteins & [36] \\
\hline Graphene oxide & Boronate & Glycoprotein & [37] \\
\hline Latex NP & Quaternary ammonium & Saccharides & [38] \\
\hline Poly DVB NP \& poly EDMA NP & DVB \& EDMA & Proteins & [39] \\
\hline Latex NP & Quaternary ammonium \& sulfonate & Proteins & [40] \\
\hline Hydroxyapatite NP & Hydroxyapatite & Phosphopeptides and protein & [41] \\
\hline $\mathrm{SiO}_{2} / \mathrm{TiO}_{2}$ & $\mathrm{SiO}_{2}$ & Phosphopeptides & [42] \\
\hline $\mathrm{SiO}_{2} / \mathrm{TiO}_{2}$ & Boronic acid & Glycoproteins & [43] \\
\hline
\end{tabular}

the monolith surface both by individual modification and interchangeable functionality between them (Figure 1) by simple chemical reactions. This new strategy has resulted in a diverse $\mathrm{Au}$ NP monolith with functional groups like $\mathrm{COOH}, \mathrm{OH}$ and $\mathrm{NH}_{2}$, which were then directly used as ligands for efficient separation of peptides (Tyr-Gly, Tyr-Gly-Gly, and Tyr-Gly-GlyPhe-Leu) and proteins (ribonuclease A, cyctochrome c, and myoglobin). It is worthwhile to note that Au NP immobilization did not affect the original permeability of the poly GMA-EDMA monolith, i.e., $0.26 \mathrm{MPa} / \mathrm{cm}$. To increase total coverage of $\mathrm{Au}$ NP's on poly GMA-EDMA monolith, they designed a three-step chemical strategy: ${ }^{[26]}(1)$ immobilization of cystamine on epoxy monolith via ring opening reaction to form cystamine-monolith; (2) treatment with tris(2-carboxylethyl) phosphine has liberated thiol groups present in cystamine to form thiol-monolith; and (3) immobilization of Au NPs on the thiol-monolith. EDS (energy dispersive X-ray spectroscopy) experimental data indicated that maximum nanoparticle loading (60.6 wt\%, no aggregation of nanoparticles) on the monolith was achieved with $40 \mathrm{~nm}$ Au NP. In this study, 1-octanethiol and 1-octadecanethiol were the ligands and were immobilized on Au NPs present on monolith. Good protein (ribonuclease A, cytochrome c, myoglobin) separation was achieved when monoliths were incorporated with $15-30 \mathrm{~nm}$ Au NP's (46.5 wt\%-58.4 wt\% load) only.

In another study, ${ }^{[27]}$ they prepared a double layered Au NP polystyrene monolithic column to enhance the hydrophilicity for separation of nucleosides. The layered assembly of Au NP's on monolith was achieved as follows: (i) The native polystyrene monolith with bromine groups was reacted with cystamine followed by tris(2-carboxylethyl) phosphine treatment. This resulted in free thiol groups on the surface; (ii) The first layer of Au NP's was deposited on the monolith surface by reacting thiol groups with Au NP's; (iii) An amine molecular layer was created by performing a reaction between the first layered Au NP's and polyethylene imine (PEI) molecules; (iv) The second Au NP's layer was finally achieved by reacting free amine groups of $\mathrm{PEI}$ with introduction of Au NP's. For separation of nucleosides (thymine, adenosine, cytidine, cytosine, and guanosine) and peptides (Phe-Gly-Phe-Gly, Val-Try-Val, Gly-Phe, Gly-Leu, GlyTry, Lys-Val, Gly-Gly-Gly), L-cysteine was used as the ligand on this two-layered Au NP fused monolith.

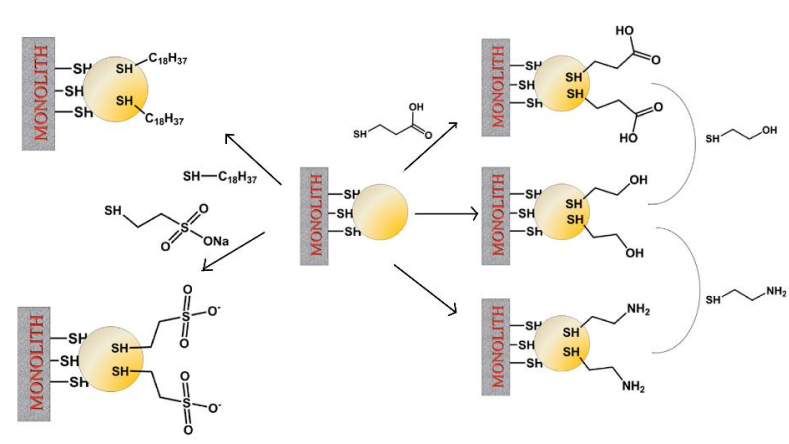

Figure 1 Schematic diagram of different chemical approaches to modify AuNP's on the monolith surface. ${ }^{[25]}$

Alwael et al. ${ }^{[28]}$ immobilized Au NP's on EDMA monolith pipette tip via Azlactone chemistry. Subsequent treatment of AuNP's with 3,3'-dithiodipropionic acid di( $N$-hydroxysuccinimide ester) resulted in terminal succinimidyl groups. Erythrina cristagalli $(E C L)$ lectin was then immobilized on the monolith and applied for enrichment of glycoprotein desialylated transferrin over non-glycoprotein ribonuclease B. The glycoprotein selectivity of this lectin Au NP-monolith pipette tip was demonstrated in Figure 2 using transferrin spiked $E$. coli cell lysate. Transferrin was only selectively enriched (decrease of peak intensity in chromatogram profile, Figure $2 B(b)$ ) from the cell lysate. Figure $2 \mathrm{~B}(\mathrm{c})$ represents the elution profile of transferrin using galactose as competitive eluting agent in elution buffer.

Lu et al. ${ }^{[29]}$ developed ionic liquid (IL)-Au NP-silica monoliths for capillay electrochromatography application. Here, IL (1-methyl-2-mercapto-3-butylimidazolium bromide) is immobilized on pre-prepared Au NP-silica monolith via the existing thio molecule in its structure. The presence of IL's on Au NP-silica monolith surface has resulted in successful separation of thiourea, uridine, guanosine, guanine, and adenine (from a mixture). These molecules are of biological significance and pharmaceutical interest. 

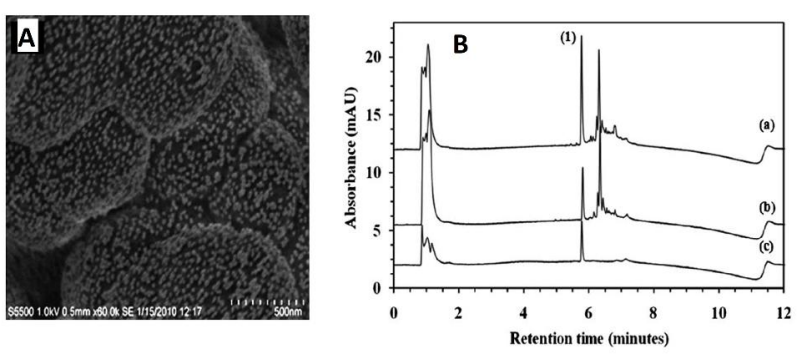

Figure 2 (A) Field emission SEM image displaying a uniform distribution of Au NP's over monolithic surface, and (B) chromatogram profile of transferrin (marked as (1)) spiked $E$. Coli cell lysate: (a) before enrichment; (b) after enrichment and (c) eluted fraction. ${ }^{[28]}$

Zhang et al. ${ }^{[30]}$ developed an efficient system to selectively enrich glycopeptides from a $5 \mu \mathrm{g}$ digested human plasma sample (without 14 high abundant proteins). Subsequently, an on-line deglycosylation of trapped glycopeptides was done during the elution process. The Au NP immobilized poly GMAPEGDA (glycidyl methacrylate-co-polyethylene glycol diacrylate) monolith was prepared according to Ref. [26] and served as a matrix for both glycopeptide enrichment and on-line deglycosylation. Replacement of EGMA as crosslinker with PEGDA has enhanced the hydrophilicity of the monolith (water contact angle was close to 0 ). For glycopeptide enrichment, cysteine was the ligand. For on-line deglycosylation, the enzyme PNGase $F$ is the ligand. In both cases, the ligands were immobilized directly on Au NPs present on the monolith surface. The use of cysteine as the ligand helped to perform the glycopeptide enrichment under weak alkaline buffer conditions (10 mM ammonium bicarbonate), which is also a compatible buffer for deglycosylation process. Overall, this study has yielded $196 \mathrm{~N}$-linked glycopeptides that corresponds to 122 glycoproteins. In another study, ${ }^{[31]}$ they immobilized a "human $\alpha$-thrombin aptamer (Apt 29) with thiol groups" on the Au NP-GMA-PEGDA monolith. The dynamic binding capacity of the monolith was $277.1 \mu \mathrm{mol} / \mathrm{L}$. This affinity monolith captured selectively "human $\alpha$-thrombin" from plasma sample spiked with 1000 fold human $\alpha$-thrombin and could run over 100 times cycles with no significant loss of capacity.

Currivan et al. ${ }^{[32]}$ designed a two zone monolith capillary system that involves (i) protein trapping by affinity chromatography principle (Au NP was used as ligand) and (ii) separation of eluted proteins by reverse phase chromatography principle. The proof-of-concept was successfully demonstrated using 4 model protein mixture (bovine serum albumin, ribonuclease $b$, insulin and carbonic anhydrase).

\section{Iron oxide Nanoparticles (IO NP)}

Krenkova and Foret ${ }^{[33]}$ immobilized iron oxide (IO) nanoparticles (average diameter of $19.08 \mathrm{~nm}$ ) on quaternary amine poly GMA-EDMA monolith. The synthesized $10 \mathrm{NP}$ is composed of $\mathrm{Fe}_{3} \mathrm{O}_{4}$ and $\gamma-\mathrm{Fe}_{2} \mathrm{O}_{3}$, and they are stable due to the presence of citrate ions. The model molecule, adenosine-5'triphosphate, showed a dynamic binding capacity of $47.4 \mathrm{mg}$ per $\mathrm{mL}$ monolith. This monolith showed selective phosphopeptide enrichment of both $\beta$ - \& $\alpha$-casein digests. For $\alpha$-casein (Figure 3), 13 significant phosphopeptides were identified in comparison to without enrichment process (5 phosphopeptides) and commercial TiO tips (5 phosphopeptides). In case of $\beta$-casein, the signal intensities of the 3 identified phosphopeptides increased significantly with the use of this monolith in comparison to without enrichment and commercial TiO tips.
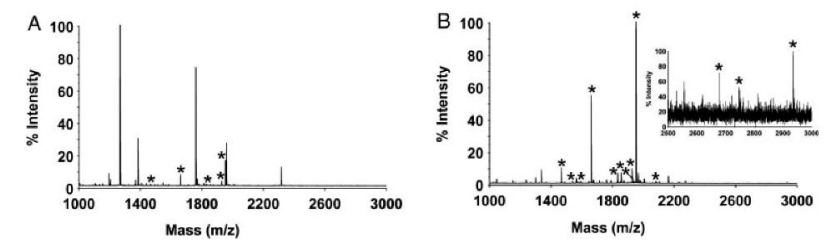

Figure 3 MALDI-ToF MS of $\alpha$-casein digest (* represents phosphopeptides) before $(A)$ and after $(B)$ enrichment with IO NP-monolith. ${ }^{[33]}$

\section{Carbon based nanotubes}

Li et al. ${ }^{[34]}$ prepared single-wall carbon nanotube (SWNT) fused poly VBC-EDMA (vinyl benzyl chloride-co-ethylene dimethracrylate) monolith in a single step in a PEI pre-functionalized silica column. PEI coating was done to generate annular EOF (electroosmotic flow). Incorporation of SWNT has significantly enhanced two properties of the monolith: (i) surface area of the monolith from 7.90 to $11.01 \mathrm{~m}^{2} / \mathrm{g}$, and (ii) EOF mobility from $1.95 \times 10^{-8}$ to $2.53 \times 10^{-8} \cdot \mathrm{m}^{2} \cdot \mathrm{V}^{-1} \cdot \mathrm{s}^{-1}$ and only a slight increase in the pore size (from 3.26 to $3.35 \mathrm{~nm}$ ). Separation of the 5 model peptides (methionine encephalin (M), leucine encephalin (L), Val-Tyr-Val (V), angiotensin II (A), and Gly-Tyr (G)) with good retention factor was achieved only if SWNT was present within the monolith structure (Figure 4). The change in the migration order of peptides $\mathrm{V}$ and $\mathrm{A}$ in presence of SWNT relates to the specific interaction of carbon nanotube with proteins and peptides.
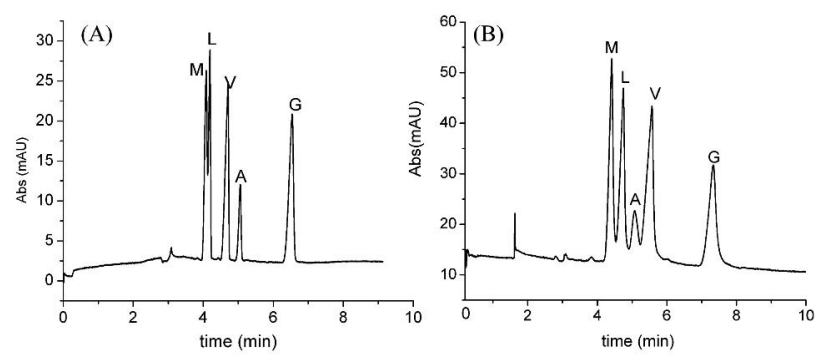

Figure 4 Electrochormatogram of five model peptide mixture in A) SWNT-poly VBC-EDMA monolith with PEI coating on silica innerwall surface and B) poly VBC-EDMA monolith with PEI coating on silica innerwall surface. ${ }^{[34]}$

To overcome the poor carbon nanotube dispersion and prevent formation of MWNT (multi-wall carbon nanotube) aggregates in polymer matrix, Zhou et al. ${ }^{[35]}$ designed an approach called as "oligomer matrix-assisted dispersion (OMAD)" to achieve homogeneous distribution of multiwall carbon nanotubes in poly MMA-EDMA monolith. The strategy involved addition of MWNT to MMA-EDMA (methyl methacrylate-co-ethylene dimethracrylate) oligomer solution followed by polymerization of the mixture for $24 \mathrm{~h}$. The surface area of the monolith has increased to 6.2 folds. At $\mathrm{pH} 6$, this negatively charged monolith showed good affinity to positively charged proteins (hemoglobin $(\mathrm{pl} 7 ; 4.4 \mathrm{mg} / \mathrm{mL}$ ) and cytochrome c ( $\mathrm{pl} 10$, $4.1 \mathrm{mg} / \mathrm{mL}$ )) and no affinity to negatively charged bovine serum albumin ( $\mathrm{pl}$ 4.6). This MWNT-poly MMA-EDMA monolith has efficiently captured a 5000 fold diluted Hemoglobin from human blood samples. Recently, ${ }^{[37]}$ they extended this work by replacing MWNT with boronate functionalized graphene oxide (GO) in MMA-EDMA monolith structure and applied it to successfully separate two glycoproteins (ovalbumin and ovotransferrin) from egg white. Incorporation of $\mathrm{GO}$ has 
tremendously increased the surface area of the monolith from 6.3 to $169.4 \mathrm{~m}^{2} / \mathrm{g}$ and without affecting the permeability. The presence of boronate on GO has enhanced the dynamic binding capacity of the monolith by 10 fold.

To enhance the protein retention and separation efficiency of an octadecyl (C18) monolith (poly ODA-TRIM, (octadecyl acrylate-co-trimethylolpropane trimethacrylate)), Mayadunne and EI Rassi ${ }^{[36]}$ incorporated MWNTs into the C18 monolith structure. MWNTs were chosen due to its hydrophobic property and the potential to interact with aromatic aminoacid's (tryptophan, phenylalanine and tyrosine) via $\pi-\pi$ bond formation. It is observed that the outer diameter of MWNTs plays an important role in enhancing the protein peak sharpness during separation, i.e., larger the diameter, better is the peak sharpness. The MWNT-C18 poly ODA-TRIM monolith has shown better protein (ribonuclease $A$, cytochrome $c$, lysozyme, transferrin, bovine serum albumin, $\beta$-lactoglobulin $A$ and ovalbumin) retention and separation efficiency than the monolith without MNWT.

\section{Polymer based nanoparticles}

Hilder et al. $^{[38]}$ fused quaternary amine functional latex nanoparticles $(60 \mathrm{~nm})$ on to a pre-synthesized poly BMAEDMA-AMPS (butyl methacrylate-co-ethylene dimethacrylateco-2-acrylamido-2-methyl-1-propanesulfonic acid) monolith via electrostatic bonding between the quaternary amine groups present on latex NPs and sulfonate groups present on the monolith surface. Incorporation of latex NPs enhanced the surface area from 35.2 to $47.3 \mathrm{~m}^{2} / \mathrm{g}$ along with a decrease of permeability, i.e., increase of backpressure from 2.6 to $5.7 \mathrm{MPa}$. A monolith with $0.97 \mu \mathrm{m}$ pore size has presented a good separation and peak resolution of 7 model saccharide mixture $(D-(+)$-galactose, $D$-(+)-glucose, $D$-(+)-xylose, $D-(+)$-mannose, maltose, $D$-(-)-fructose, sucrose). With the optimized $\mathrm{pH}$ and buffer condition, the three saccharides (maltotriose, maltose, and glucose) present in enzymatically processed corn starch were separated, while maltotriose was strongly adsorbed on the column and therefore could not elute from the column.

The group of Prof. Hilder studied the influence of neutral $\left(50-200 \mathrm{~nm}\right.$ poly DVB and 5-350 nm poly EDMA $\left.{ }^{[39]}\right)$ and charged latex NPs (both positive $\left(\mathrm{NR}_{4}{ }^{+}, \mathrm{AS} 17\right.$ \& PA100) and negative $\left.\left(\mathrm{SO}_{3}{ }^{-}\right)^{[40]}\right)$ incorporation within the confinements of PEGDA cryogel monolith. PEGDA (Mn 258) is chosen as the single monomer for monolith fabrication due to its two vital properties: (i) water solubility and (ii) contains both hydrophilic and hydrophobic molecular sites that are essential for hydrophobic interaction chromatography (HIC). In both studies, NPs were added to the polymer mixture prior to its polymerization at sub-zero temperatures (i.e., cryo polymerization). In case of neutral NPs monolith system, NPs were distributed heterogeneously and within the inner cavities of the polymer matrix. Whereas with charged NPs, they were predominantly present at the polymer surface. This difference in NP distribution relates to the difference in the polarity, surface charge and their segregation in the interfaces during polymerization process. Neutral NPs-monolith's (poly DVB NP-monolith \& poly EDMA NP-monolith) separated the four model proteins (myoglobin, ribonuclease A, lysozyme and $\alpha$-chymotrypsinogen A) using hydrophobic interaction chromatography. ${ }^{[39]}$ The overall protein separation was good in both cases, but high amount of poly EDMA NPs are needed to achieve similar separation performance as the poly DVB NPmonolith. This is due to the slight differences in the hydrophobic nature of both NPs. In case of charged NP incorporated monoliths, absence of NPs led to unsuccessful separation of lysozyme and isoform of $\alpha$-chymotrypsinogen $\mathrm{A}$ in the protein mixture. Addition of charged NPs $\left(\left(\mathrm{NR}_{4}{ }^{+}\right)\right.$and $\left.\left.\mathrm{SO}_{3}{ }^{-}\right)\right)$in the cryogel has enhanced the peak width and peak symmetry along with good separation of all proteins present in the mixture. It is important to note that lysozyme, the highest isoelectric point (pl) protein of all, was strongly bound to the $\mathrm{SO}_{3}{ }^{-}$monolith and was not eluted under the applied eluting conditions. This indicates the strong affinity of lysozyme to $\mathrm{SO}_{3}{ }^{-}$groups present on polymer surface.

\section{Hydroxyapatite (HA) nanoparticle}

Krenkova et al..$^{[41]}$ incorporated rod shaped HA NPs (average size of $50 \times 150 \mathrm{~nm}$, surface area of $100 \mathrm{~m}^{2} / \mathrm{g}$ ) in poly HEMA-EDMA monolith prior to in situ polymerization. As HA contains both calcium (positive charge) and phosphate (negative charge) molecules, immobilization after monolith polymerization can lead to aggregates. Due to this reason, HA NPs were incorporated to polymer solution followed by polymerization of the composite mixture. With increasing amount of HA NP loading in the monolith, the protein separation (ovalbumin, myoglobin, lysozyme, and cytochrome c) improved but with a significant increase in flow resistance. This HA NP-poly HEMA-EDMA monolith application was studied in two cases: (i) separation of monoclonal antibody complex and (ii) enrichment of phosphopeptides from tryptic digest of $\beta$-casein, $\alpha$-casein and ovalbumin. Overall, this enrichment approach has identified 3 phosphopeptides for $\beta$-casein (Figure 5B); 11 phosphopeptides for $\alpha$-casein (Figure 5D); and 2 phosphopeptides for ovalbumin. Whereas, when analyzed without enrichment process 0 phosphopeptides for $\beta$-casein (Figure 5A); 2 phosphopeptides for $\alpha$-casein (Figure $5 \mathrm{C}$ ); and 0 phosphopeptides for ovalbumin were identified by MALDI-MS. These results showcase the importance of enrichment process prior to MS analysis.
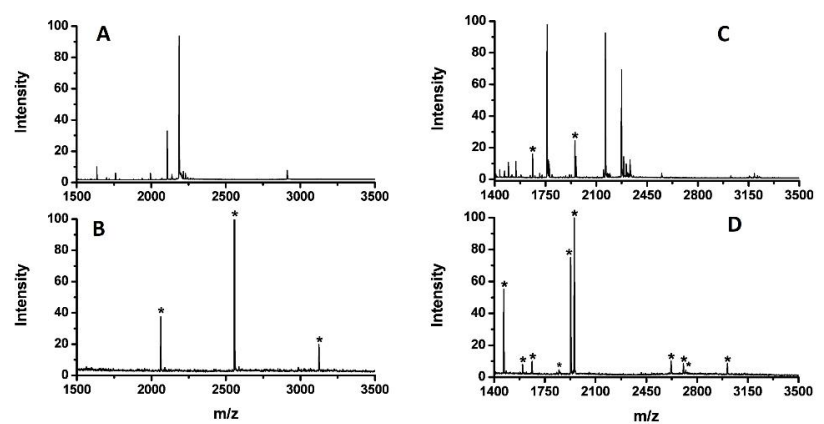

Figure 5 MALDI-MS of tryptic digest of $\beta$-casein (A: before and B: after enrichment), and $\alpha$-casein (C: before and D: after enrichment). ${ }^{[41] *}$ Phosphopeptides.

\section{Inorganic nanocomposites}

Wang et al. ${ }^{[42]}$ synthesized $\mathrm{SiO}_{2} / \mathrm{TiO}_{2}$ composite monolith (narrow pore size of $3.6 \mathrm{~nm}$, surface area of $115 \mathrm{~m}^{2} / \mathrm{g}$ ) via sol-gel technology and employed $\mathrm{SiO}_{2} / \mathrm{TiO}_{2}$ composite as ligand for enrichment of phosphopeptides from human serum, non-fat milk and egg white. For example, 11 phosphopeptides were identified from milk samples using $\mathrm{SiO}_{2} / \mathrm{TiO}_{2}$ composite in comparison to 5 phosphopeptides using $\mathrm{TiO}_{2}$ material itself and 3 without the use of any material. The obtained data (MS, Figure 6) on human serum sample suggest the impact of $\mathrm{SiO}_{2}$ molecules in the composite. 
General Chemistry
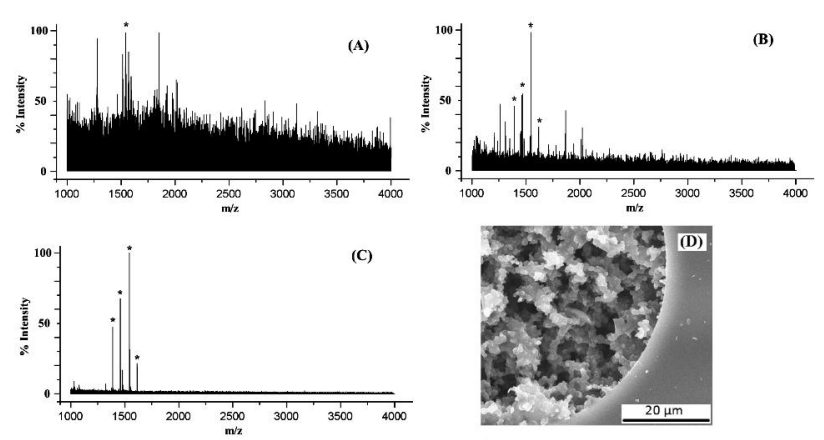

Figure 6 MS data of Human serum: (A) direct analysis (without enrichment process); (B) enriched using $\mathrm{TiO}_{2}$ material; and $(\mathrm{C})$ enriched using $\mathrm{SiO}_{2}-\mathrm{TiO}_{2}$ composite monolith. SEM image of $\mathrm{SiO}_{2}-\mathrm{TiO}_{2}$ composite monolith (D). ${ }^{[42]}$ * Phophopeptides.

Yang et al. ${ }^{[43]}$ prepared a $\mathrm{SiO}_{2} / \mathrm{TiO}_{2}$ composite monolithic column functionalized with boronate's in a single step. Here, boronate acts as the ligand to specifically target glycoproteins. The monolith had a well interconnected porous network, pore size of $4.76 \mathrm{~nm}$ and a permeability $\left(K_{\mathrm{P}, \mathrm{F}}=32.3 \times 10^{-14} \mathrm{~m}^{2}\right)$, which is 13 times higher than the conventional packed columns $\left.\left(2.5 \times 10^{-14} \mathrm{~m}^{2}\right)\right)$. The monolith showed excellent specificity to model glycoproteins (ovalbumin and lactoferrin) over nonglycoproteins (bovine serum albumin, myoglobin, and cytochrome $\mathrm{c}$ at near physiological conditions (phosphate buffer $\mathrm{pH}$ 7 ). Its affinity to both human and mouse monoclonal antibodies was also demonstrated.

\section{Conclusions}

Overall 20 research articles have been published since the inception of nanomaterials within the monolith microcolumns to enhance the separation of biomolecules. The separation of biomolecules was achieved using affinity chromatography, ion exchange chromatography, hydrophilic interaction chromatography, hydrophobic interaction chromatography and in some cases a combination of these chromatography principles. In case of nanomaterials, Au NPs (9 research articles) and carbon based nanomaterials ( 4 research articles) have been explored more than other nanomaterials. The obtained biomolecule separation using nanomaterials within the confinements of monoliths is highly encouraging. The use of nanomaterials in monolithic microcolumns is progressing slowly than what was expected in the early 2000's. We expect the carbon based nanomaterials will be exploited further due to the current trend in the field of nanomaterials and their diverse properties. Also, we can expect a combination of two or more nanomaterials within the monolith to further enhance the separation performance.

\section{Acknowledgement}

Dr. K. K. R. Tetala acknowledges SERB-DST for funding under the Young Scientist Scheme (No. SB/YS/LS-160/2014).

\section{References}

[1] Scopes, R. K. Protein Purification-Principles and Practice, Springer-Verlaag, New York, 1994, p. 380.

[2] Smejkal, G. B.; Lazarev, A. Separation Methods in Proteomics, CRC Press Taylor \& Francis Group, Florida, 2006, p. 536.

[3] Hermanson, G. T.; Krishna Mallia, A.; Smith, P. K. Immobilized
Affinity Ligand Techniques, In Immobilized Affinity Ligand Techniques, Academic Press, San Diego, California, 1992, p. 51-275.

[4] Przybycien, T. M.; Pujar, N. S.; Steele, L. M. Curr. Opin. Biotechnol. 2004, 15, 469.

[5] Hjerten, S.; Liao, J. L.Zhang, R., J. Chromatogr. 1989, 473, 273.

[6] Svec, F.; Frechet, J. M. J. Anal. Chem. 1992, 64, 820.

[7] Tetala, K. K. R.; van Beek, T. A. J. Sep. Sci. 2010, 33, 422.

[8] Tetala, K. K. R.; Vijayalakshmi, M. A. Anal. Chim. Acta 2016, 906, 7.

[9] Ghanem, A.; Ikegami, T. J. Sep. Sci. 2011, 34, 1945.

[10] Pfaunmiller, E. L.; Paulemond, M. L.; Dupper, C. M.; Hage, D. S. Anal. Bioanal. Chem. 2013, 405, 2133.

[11] Siouffi, A. M. J. Chromatogr. A 2003, 1000, 801.

[12] Strancar, A.; Podgornik, A.; Barut, M.; Necina, R. Adv. Biochem. Eng. Biotechnol. 2002, 76, 49

[13] Svec, F. J. Sep. Sci. 2004, 27, 747.

[14] Svec, F. J. Chromatogr. A 2012, 1228, 250.

[15] Svec, F.; Huber, C. G. Anal. Chem. 2006, 78, 2100

[16] Speltini, A.; Merli, D.; Profumo, A. Anal. Chim. Acta 2013, 783, 1.

[17] Zhang, Z.; Wang, Z.; Liao, Y.; Liu, H. J. Sep. Sci. 2006, 29, 1872.

[18] Ahmadi, M.; Elmongy, H.; Madrakian, T.; Abdel-Rehim, M. Anal. Chim. Acta 2017, 958, 1

[19] Duan, A.-H.; Xie, S.-M.; Yuan, L.-M. Trends Anal. Chem. 2011, 30, 484.

[20] Mahouche-Chergui, S.; Guerrouache, M.; Carbonnier, B.; Chehimi, M. M. Colloids Surf. A. Physicochem. Eng. Asp. 2013, 439, 43.

[21] Zhang, M.; Qiu, H. Trends Anal. Chem. 2015, 65, 107.

[22] Tong, S.; Liu, S.; Wang, H.; Jia, Q. Chromatographia 2014, 77, 5.

[23] Damian, C.; Sinead, C.; Brett, P. Proteomics 2012, 12, 2904.

[24] Xu, Y.; Cao, Q.; Svec, F.; Fréchet, J. M. J. Anal. Chem. 2010, 82, 3352.

[25] Cao, Q.; Xu, Y.; Liu, F.; Svec, F.; Frechet, J. M. Anal. Chem. 2010, 82, 7416.

[26] Lv, Y.; Alejandro, F. M.; Frechet, J. M.; Svec, F. J. Chromatogr. A 2012, 1261, 121

[27] Lv, Y.; Lin, Z.; Svec, F. Anal. Chem. 2012, 84, 8457.

[28] Alwael, H.; Connolly, D.; Clarke, P.; Thompson, R.; Twamley, B.; O'Connor, B.; Paull, B. Analyst 2011, 136, 2619.

[29] Lu, J.; Ye, F.; Zhang, A.; Chen, X.; Wei, Y.; Zhao, S. Analyst 2012 137, 5860.

[30] Liang, Y.; Wu, C.; Zhao, Q.; Wu, Q.; Jiang, B.; Weng, Y.; Liang, Z.; Zhang, L.; Zhang, Y. Anal. Chim. Acta 2015, 900, 83.

[31] Chen, Y.; Deng, N.; Wu, C.; Liang, Y.; Jiang, B.; Yang, K.; Liang, Z.; Zhang, L.; Zhang, Y. Talanta 2016, 154, 555.

[32] Currivan, S.; Connolly, D.; Paull, B. Microchem. J. 2013, 111, 32.

[33] Krenkova, J.; Foret, F. J. Sep. Sci. 2011, 34, 2106.

[34] Li, Y.; Chen, Y.; Xiang, R.; Ciuparu, D.; Pfefferle, L. D.; Horváth, C.; Wilkins, J. A. Anal. Chem. 2005, 77, 1398.

[35] Zhou, C.; Du, Z.; Li, G.; Zhang, Y.; Cai, Z. Analyst 2013, 138, 5783.

[36] Mayadunne, E.; Rassi, Z. E. Talanta 2014, 565.

[37] Zhou, C.; Chen, X.; Du, Z.; Li, G.; Xiao, X.; Cai, Z. J. Chromatogr. A 2017, 1498, 90.

[38] Hilder, E. F.; Svec, F.; Fréchet, J. M. J. J. Chromatogr. A 2004, 1053, 101.

[39] Arrua, R. D.; Nordborg, A.; Haddad, P. R.; Hilder, E. F. J. Chromatogr. A 2013, 1273, 26.

[40] Arrua, R. D.; Haddad, P. R.; Hilder, E. F. J. Chromatogr. A 2013, 1311,121

[41] Krenkova, J.; Lacher, N. A.; Svec, F. Anal. Chem. 2010, 82, 8335.

[42] Wang, S. T.; Wang, M. Y.; Su, X.; Yuan, B. F.; Feng, Y. Q. Anal. Chem. 2012, 84, 7763.

[43] Yang, Q.; Huang, D.; Zhou, P. Analyst 2014, 139, 987.

Received May 11, 2018

Accepted June 17, 2018 